\title{
Precision measurements of $B\left(D^{+} \rightarrow \mu^{+} \nu_{\mu}\right)$, the pseudoscalar decay constant $f_{D^{+}}$, and the quark mixing matrix element $\left|V_{\text {cd }}\right|$
}

M. Ablikim, ${ }^{1}$ M. N. Achasov ${ }^{8, b}$ X. C. Ai, ${ }^{1}$ O. Albayrak, ${ }^{4}$ D. J. Ambrose, ${ }^{41}$ F. F. An, ${ }^{1}$ Q. An, ${ }^{42}$ J. Z. Bai, ${ }^{1}$ R. Baldini Ferroli, ${ }^{19 a}$ Y. Ban, ${ }^{28}$ J. V. Bennett, ${ }^{18}$ M. Bertani, ${ }^{19 a}$ J. M. Bian, ${ }^{40}$ E. Boger, ${ }^{21, c}$ O. Bondarenko, ${ }^{22}$ I. Boyko, ${ }^{21}$ S. Braun, ${ }^{37}$ R. A. Briere, ${ }^{4}$ H. Cai,${ }^{47}$ X. Cai, ${ }^{1}$ O. Cakir ${ }^{36 a}$ A. Calcaterra, ${ }^{19 a}$ G. F. Cao, ${ }^{1}$ S. A. Cetin, ${ }^{36 b}$ J. F. Chang, ${ }^{1}$ G. Chelkov, ${ }^{21, c}$ G. Chen, ${ }^{1}$ H. S. Chen, ${ }^{1}$ J. C. Chen, ${ }^{1}$ M. L. Chen, ${ }^{1}$ S. J. Chen ${ }^{26}{ }^{26}$ X. Chen, ${ }^{1}$ X. R. Chen, ${ }^{23}$ Y. B. Chen, ${ }^{1}$ H. P. Cheng, ${ }^{16}$ X. K. Chu, ${ }^{28}$ Y. P. Chu, ${ }^{1}$ D. Cronin-Hennessy, ${ }^{40}$ H. L. Dai, ${ }^{1}$ J. P. Dai, ${ }^{1}$ D. Dedovich,${ }^{21}$ Z. Y. Deng, ${ }^{1}$ A. Denig, ${ }^{20}$ I. Denysenko, ${ }^{21}$ M. Destefanis, ${ }^{45 a, 45 c}$ W. M. Ding, ${ }^{30}$ Y. Ding ${ }^{24}$ C. Dong, ${ }^{27}$ J. Dong, ${ }^{1}$ L. Y. Dong, ${ }^{1}$ M. Y. Dong, ${ }^{1}$ S. X. Du, ${ }^{49}$ J. Z. Fan, ${ }^{35}$ J. Fang, ${ }^{1}$ S. S. Fang, ${ }^{1}$ Y. Fang, ${ }^{1}$ L. Fava, ${ }^{45 b, 45 c}$ C. Q. Feng, ${ }^{42}$ C. D. Fu, ${ }^{1}$ J. L. Fu, ${ }^{26}$ O. Fuks, ${ }^{21, b}$ Q. Gao, ${ }^{1}$ Y. Gao, ${ }^{35}$ C. Geng, ${ }^{42}$ K. Goetzen, ${ }^{9}$ W. X. Gong, ${ }^{1}$ W. Gradl,${ }^{20}$ M. Greco, ${ }^{45 a, 45 c}$ M. H. Gu, ${ }^{1}$ Y. T. Gu, ${ }^{11}$ Y. H. Guan, ${ }^{1}$ A. Q. Guo ${ }^{27}$ L. B. Guo ${ }^{25}$ T. Guo, ${ }^{25}$ Y. P. Guo ${ }^{27}$ Y. P. Guo ${ }^{20}{ }^{2}$ Y. L. Han,${ }^{1}$ F. A. Harris ${ }^{39}$ K. L. He, ${ }^{1}$ M. He, ${ }^{1}$ Z. Y. He, ${ }^{27}$ T. Held ${ }^{3}{ }^{\text {Y. K. Heng, }}{ }^{1}$ Z. L. Hou, ${ }^{1}$ C. Hu,${ }^{25}$ H. M. Hu, ${ }^{1}$ J. F. Hu, ${ }^{37}$ T. Hu, ${ }^{1}$ G. M. Huang, ${ }^{5}$ G. S. Huang, ${ }^{42}$ J. S. Huang, ${ }^{14}$ L. Huang, ${ }^{1}$ X. T. Huang, ${ }_{16}$ Y. Huang ${ }_{1}^{26}$ T. Hussain, ${ }^{44}$ C. S. Ji, ${ }^{42}$ Q. Ji, ${ }^{1}$ Q. P. Ji, ${ }^{27}$ X. B. Ji, ${ }^{1}$ X. L. Ji, ${ }^{1}$ L. L. Jiang,,${ }^{1}$ X. S. Jiang, ${ }^{1}$ J. B. Jiao, ${ }^{30}$ Z. Jiao, ${ }^{16}$ D. P. Jin, ${ }^{1}$ S. Jin, ${ }^{1}$ T. Johansson, ${ }^{46}$ N. Kalantar-Nayestanaki, ${ }^{22}$ X. L. Kang, ${ }^{1}$ X. S. Kang, ${ }^{27}$ M. Kavatsyuk, ${ }^{22}$ B. Kloss, ${ }^{20}$ B. Kopf, ${ }^{3}$ M. Kornicer, ${ }^{39}$ W. Kuehn, ${ }^{37}$ A. Kupsc, ${ }^{46}$ W. Lai, ${ }^{1}$ J. S. Lange,${ }^{37}$ M. Lara, ${ }^{18}$ P. Larin, ${ }^{13}$

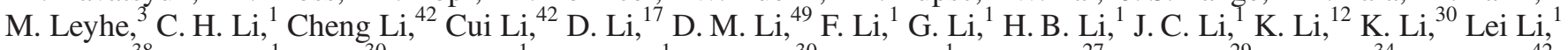
P. R. Li, ${ }^{38}$ Q. J. Li, ${ }^{1}$ T. Li, ${ }^{30}$ W. D. Li, ${ }^{1}$ W. G. Li, ${ }^{1}$ X.L. Li, ${ }^{30}$ X. N. Li, ${ }^{1}$ X. Q. Li, ${ }^{27}$ X. R. Li, ${ }^{29}$ Z. B. Li, ${ }^{34}$ H. Liang, ${ }^{42}$ Y. F. Liang, ${ }^{32}$ Y. T. Liang, ${ }^{37}$ D. X. Lin,${ }^{13}$ B. J. Liu, ${ }^{1}$ C. L. Liu, ${ }^{4}$ C. X. Liu, ${ }^{1}$ F. H. Liu, ${ }^{31}$ Fang Liu, ${ }^{1}$ Feng Liu,${ }^{5}$ H. B. Liu, ${ }^{11}$ H. H. Liu, ${ }^{15}$ H. M. Liu, ${ }^{1}$ J. Liu, ${ }^{1}$ J. P. Liu ${ }^{47}$ K. Liu,${ }^{35}$ K. Y. Liu, ${ }^{24}$ P. L. Liu, ${ }^{30}$ Q. Liu, ${ }^{38}$ S. B. Liu, ${ }^{42}$ X. Liu, ${ }^{23}$ Y. B. Liu ${ }^{27}$ Z. A. Liu, ${ }^{1}$ Zhiqiang Liu, ${ }^{1}$ Zhiqing Liu, ${ }^{20}$ H. Loehner, ${ }^{22}$ X. C. Lou, ${ }^{1, d}$ G. R. Lu, ${ }^{14}$ H. J. Lu, ${ }^{16}$ H. L. Lu, ${ }^{1}$ J. G. Lu, ${ }^{1}$ X. R. Lu, ${ }^{38}$ Y. Lu, ${ }^{1}$ Y. P. Lu, ${ }^{1}$ C. L. Luo, ${ }^{25}$ M. X. Luo, ${ }^{48}$ T. Luo, ${ }^{39}$ X. L. Luo ${ }_{1}^{1}$ M. Lv, ${ }^{1}$ F. C. Ma, ${ }^{24}$ H. L. Ma, ${ }^{1}$ Q. M. Ma, ${ }^{1}$ S. Ma, ${ }^{1}$ T. Ma, ${ }^{1}$ X. Y. Ma, ${ }^{1}$ F. E. Maas, ${ }^{13}$ M. Maggiora, ${ }^{45,45 c}$ Q. A. Malik, ${ }^{44}$ Y. J. Mao, ${ }^{28}$ Z. P. Mao, ${ }^{1}$ J. G. Messchendorp, ${ }^{22}$ J. Min, ${ }^{1}$ T. J. Min, ${ }^{1}$ R. E. Mitchell, ${ }^{18}$ X. H. Mo, ${ }^{1}$ Y. J. Mo, ${ }^{5}$ H. Moeini, ${ }^{22}$ C. Morales Morales, ${ }^{13}$ K. Moriya, ${ }^{18}$ N. Yu. Muchnoi, ${ }^{8, a}$ H. Muramatsu, ${ }^{40}$ Y. Nefedov, ${ }^{21}$ I. B. Nikolaev, ${ }^{8, a}$ Z. Ning, ${ }^{1}$ S. Nisar, ${ }^{7}$ X. Y. Niu, ${ }^{1}$ S. L. Olsen, ${ }^{29}$ Q. Ouyang, ${ }^{1}$ S. Pacetti, ${ }^{19 b}$ M. Pelizaeus ${ }^{3}$ H. P. Peng,${ }^{42}$ K. Peters, ${ }^{9}$ J. L. Ping ${ }^{25}$ R. G. Ping, ${ }^{1}$ R. Poling, ${ }^{40}$ E. Prencipe, ${ }^{20}$ M. Qi ${ }^{26}$ S. Qian, ${ }^{1}$ C. F. Qiao, ${ }^{38}$ L. Q. Qin ${ }^{30}{ }^{\text {X. S. Qin }}{ }^{1}$ Y. Qin, ${ }^{28}$ Z. H. Qin, ${ }^{1}$ J. F. Qiu, ${ }^{1}$ K. H. Rashid, ${ }^{44}$ C. F. Redmer, ${ }^{20}$ M. Ripka, ${ }^{20}$ G. Rong, ${ }^{1, a}$ X. D. Ruan, ${ }^{11}$ A. Sarantsev, ${ }^{21, e}$ K. Schoenning, ${ }^{46}$ S. Schumann, ${ }^{20}$ W. Shan, ${ }^{28}$ M. Shao, ${ }^{42}$ C. P. Shen, ${ }^{2}$ X. Y. Shen, ${ }^{1}$ H. Y. Sheng, ${ }^{1}$ M. R. Shepherd, ${ }^{18}$ W. M. Song, ${ }_{42}^{1}$ X. Y. Song, ${ }_{32}$ S. Spataro, ${ }^{45 a, 45 c}$ B. Spruck, ${ }^{37}$ G. X. Sun, ${ }_{1}^{1}$ J. F. Sun, ${ }_{14}$ S. S. Sun ${ }^{1}$ Y. J. Sun ${ }_{32}^{46}$ Y. Z. Sun, ${ }^{1}$ Z. J. Sun, ${ }^{1}$ Z. T. Sun, ${ }^{42}$ C. J. Tang, ${ }^{32}$ X. Tang, ${ }^{1}$ I. Tapan, ${ }^{36 c}$ E. H. Thorndike, ${ }^{41}$ D. Toth,${ }^{40}$ M. Ullrich,${ }^{37}$ I. Uman, ${ }^{36 b}$ G. S. Varner, ${ }^{39}$ B. Wang, ${ }^{27}$ D. Wang ${ }^{28}$ D. Y. Wang, ${ }^{28}$ K. Wang, ${ }^{1}$ L. L. Wang, ${ }^{1}$ L. S. Wang, ${ }^{1}$ M. Wang, ${ }^{30}$ P. Wang, ${ }^{1}$ P. L. Wang, ${ }_{1}^{1}$ Q. J. Wang, ${ }^{1}$ S. G. Wang, ${ }^{28}$ W. Wang, ${ }^{1}$ X. F. Wang ${ }^{35}$ Y. D. Wang, ${ }^{19 a}{ }^{20}$ Y. F. Wang, ${ }^{1}$ Y. Q. Wang, ${ }^{20}$ Z. Wang, ${ }^{1}$ Z. G. Wang, ${ }^{1}$ Z. H. Wang, ${ }^{42}$ Z. Y. Wang, ${ }^{1}$ D. H. Wei, ${ }^{10}$ J. B. Wei, ${ }^{28}$ P. Weidenkaff, ${ }^{20}$ S. P. Wen, ${ }^{1}$ M. Werner, ${ }^{37}$ U. Wiedner, ${ }^{3}$ M. Wolke, ${ }^{46}$ L. H. Wu, ${ }^{1}$ N. Wu, ${ }^{1}$ W. Wu, ${ }^{27}$ Z. Wu, ${ }^{1}$ L. G. Xia ${ }^{35}$ Y. Xia,${ }^{17}$ D. Xiao, ${ }^{1}$ Z. J. Xiao, ${ }^{25}$ Y. G. Xie, ${ }^{1}$ Q. L. Xiu, ${ }^{1}$ G. F. Xu, ${ }^{1}$ L. Xu, ${ }^{1}$ Q. J. Xu, ${ }^{12}$ Q. N. Xu ${ }^{38}$ X. P. Xu ${ }^{33}$ Z. Xue, ${ }^{1}$ L. Yan, ${ }^{42}$ W. B. Yan, ${ }^{42}$ W. C. Yan, ${ }^{42}$ Y. H. Yan, ${ }^{17}$ H. X. Yang, ${ }^{1}$ Y. Yang, ${ }^{5}$ Y. X. Yang, ${ }^{10}$ H. Ye, ${ }^{1}$ M. Ye, ${ }^{1}$ M. H. Ye, ${ }^{6}$ B. X. Yu, ${ }^{1}$ C. X. Yu, ${ }^{27}$ H. W. Yu, ${ }^{28}$ J. S. Yu, ${ }^{23}$ S. P. Yu ${ }^{30}$ C. Z. Yuan, ${ }^{1}$ W. L. Yuan, ${ }^{26}$ Y. Yuan, ${ }^{1}$ A. A. Zafar, ${ }^{44}$ A. Zallo, ${ }^{19 a}$ S. L. Zang, ${ }^{26}$ Y. Zeng, ${ }^{17}$ B. X. Zhang, ${ }^{1}$ B. Y. Zhang, ${ }^{1}$ C. Zhang, ${ }_{1}^{26}$ C. B. Zhang, ${ }_{17}$ C. C. Zhang, ${ }^{1}$ D. H. Zhang, ${ }^{1}$ H. H. Zhang, ${ }^{34}$ H. Y. Zhang, ${ }^{1}$ J. J. Zhang, ${ }^{1}$ J. L. Zhang, ${ }^{1}$ J. Q. Zhang, ${ }^{1}$ J. W. Zhang, ${ }^{1}$ J. Y. Zhang, ${ }^{1}$ J. Z. Zhang, ${ }^{1}$ S. H. Zhang, ${ }^{1}$ X. J. Zhang, ${ }_{1}^{1}$ X. Y. Zhang, ${ }^{30}$ Y. Zhang, ${ }^{1}$ Y. H. Zhang, ${ }^{1}$ Z. H. Zhang, ${ }^{5}$ Z. P. Zhang, ${ }^{42}$ Z. Y. Zhang, ${ }_{1}^{47}$ G. Zhao, ${ }^{1}$ J. W. Zhao, ${ }^{1}$ Lei Zhao, ${ }^{42}$ Ling Zhao, ${ }^{1}$ M. G. Zhao, ${ }^{27}$ Q. Zhao, ${ }^{1}$ Q. W. Zhao,

S. J. Zhao, ${ }^{49}$ T. C. Zhao, ${ }^{1}$ X. H. Zhao, ${ }^{26}$ Y. B. Zhao, ${ }^{1}$ Z. G. Zhao, ${ }^{42}$ A. Zhemchugov, ${ }^{21, b}$ B. Zheng, ${ }^{43}$ J. P. Zheng, ${ }^{1}$ Y. H. Zheng, ${ }^{38}$ B. Zhong, ${ }^{25}{ }^{25}$. Zhou, ${ }^{1}$ Li Zhou, ${ }^{27}$ X. Zhou, ${ }^{47}$ X. K. Zhou, ${ }^{38}$ X. R. Zhou, ${ }^{42}$ X. Y. Zhou, ${ }^{1}$ K. Zhu, ${ }^{1}$ K. J. Zhu, ${ }^{1}$ X. L. Zhu, ${ }^{35}$ Y. C. Zhu, ${ }^{42}$ Y. S. Zhu, ${ }^{1}$ Z. A. Zhu, ${ }^{1}$ J. Zhuang, ${ }^{1}$ B. S. Zou, ${ }^{1}$ and J. H. Zou ${ }^{1}$

\section{(BESIII Collaboration)}

\author{
${ }^{1}$ Institute of High Energy Physics, Beijing 100049, People's Republic of China \\ ${ }^{2}$ Beihang University, Beijing 100191, People's Republic of China \\ ${ }^{3}$ Bochum Ruhr-University, D-44780 Bochum, Germany \\ ${ }^{4}$ Carnegie Mellon University, Pittsburgh, Pennsylvania 15213, USA \\ ${ }^{5}$ Central China Normal University, Wuhan 430079, People's Republic of China \\ ${ }^{6}$ China Center of Advanced Science and Technology, Beijing 100190, People's Republic of China \\ ${ }^{7}$ COMSATS Institute of Information Technology, Lahore, Defence Road, \\ Off Raiwind Road, 54000 Lahore, Pakistan \\ ${ }^{8}$ G. I. Budker Institute of Nuclear Physics SB RAS (BINP), Novosibirsk 630090, Russia \\ ${ }^{9}$ GSI Helmholtzcentre for Heavy Ion Research GmbH, D-64291 Darmstadt, Germany
}




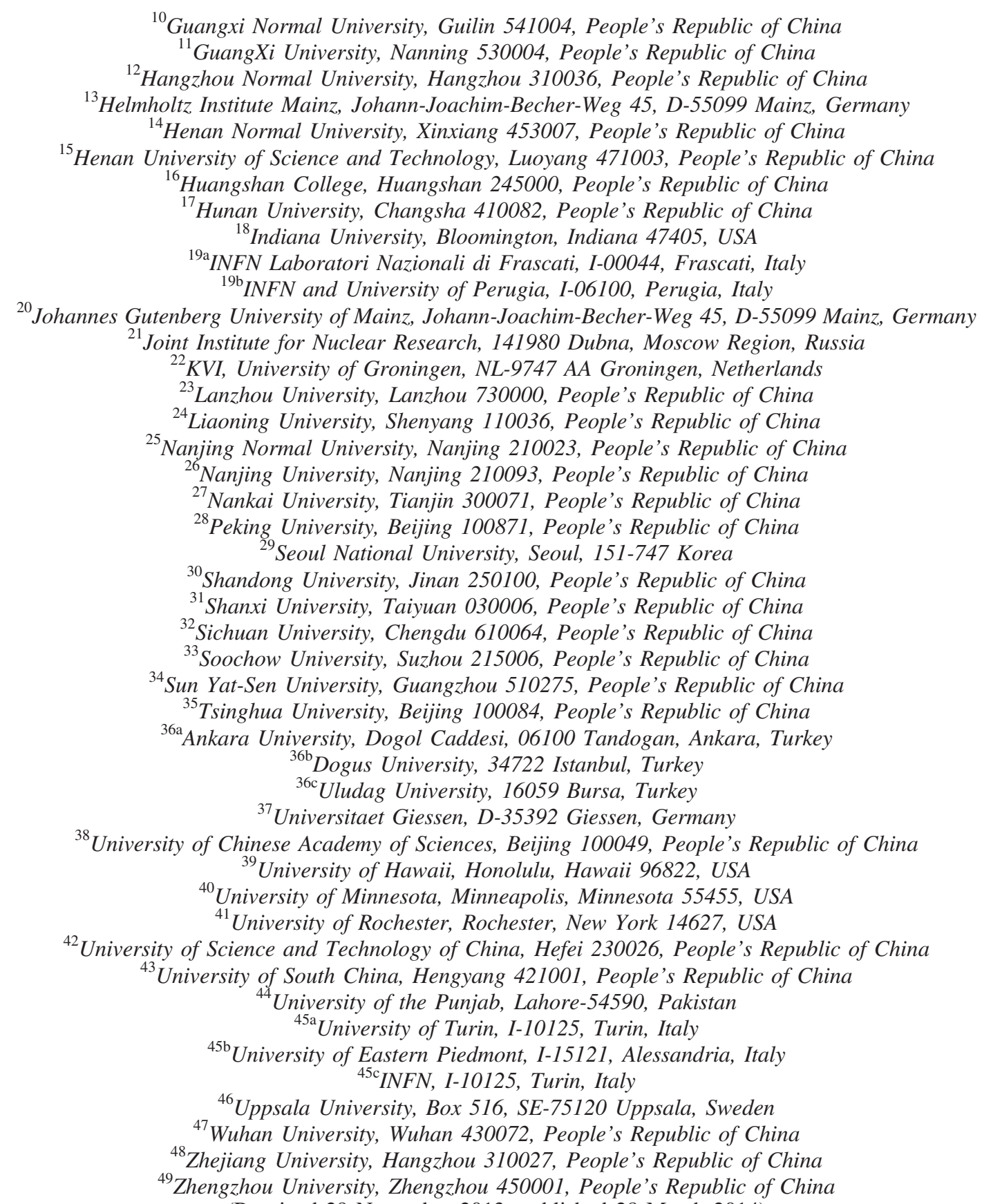

(Received 29 November 2013; published 28 March 2014)

We report a measurement of the branching fraction $B\left(D^{+} \rightarrow \mu^{+} \nu_{\mu}\right)=[3.71 \pm 0.19$ (stat $) \pm 0.06$ (sys) $] \times$ $10^{-4}$ based on $2.92 \mathrm{fb}^{-1}$ of data accumulated at $\sqrt{s}=3.773 \mathrm{GeV}$ with the BESIII detector at the BEPCII collider. This measurement, in conjunction with the Cabibbo-Kobayashi-Maskawa matrix element $\left|V_{\text {cd }}\right|$ determined from a global Standard Model fit, implies a value for the weak decay constant $f_{D^{+}}=(203.2 \pm 5.3 \pm 1.8) \mathrm{MeV}$. Additionally, using this branching fraction measurement together with a lattice QCD prediction for $f_{D^{+}}$, we find $\left|V_{\mathrm{cd}}\right|=0.2210 \pm 0.0058 \pm 0.0047$. In either case, these are the most precise results for these quantities to date.

DOI: 10.1103/PhysRevD.89.051104

PACS numbers: 13.20.Fc, 12.15.Hh, 12.38.Qk, 13.66.Bc

\footnotetext{
rongg@ihep.ac.cn

${ }^{\mathrm{b}}$ Also at Novosibirsk State University, Novosibirsk, 630090, Russia.

${ }^{c}$ Also at Moscow Institute of Physics and Technology, Moscow 141700, Russia.

${ }^{\mathrm{d}}$ Also at University of Texas at Dallas, Richardson, TX 75083, USA.

${ }^{\mathrm{e}}$ Also at PNPI, Gatchina 188300, Russia.
} 
In the Standard Model (SM) of particle physics, the $D^{+}$ meson can decay into $\ell^{+} \nu_{\ell}$ (where $\ell=e, \mu$, or $\tau$ ) via annihilation mediated by a virtual $W^{+}$boson. (Throughout this paper, the inclusion of charge conjugate channels is implied.) The decay rate depends upon the wave function overlap of the two quarks at the origin, which is parametrized by the $D^{+}$decay constant, $f_{D^{+}}$. All of the strong interaction effects between the two initial-state quarks are absorbed into $f_{D^{+}}$. In the $\mathrm{SM}$, the decay width is given by [1]

$$
\Gamma\left(D^{+} \rightarrow \ell^{+} \nu_{\ell}\right)=\frac{G_{F}^{2} f_{D^{+}}^{2}}{8 \pi}\left|V_{\mathrm{cd}}\right|^{2} m_{\ell}^{2} m_{D^{+}}\left(1-\frac{m_{\ell}^{2}}{m_{D^{+}}^{2}}\right)^{2},
$$

where $G_{F}$ is the Fermi coupling constant, $V_{\text {cd }}$ is the $c \rightarrow d$ Cabibbo-Kobayashi-Maskawa (CKM) matrix element [2], $m_{\ell}$ is the lepton mass, and $m_{D^{+}}$is the $D^{+}$-meson mass.

The decay constants $f_{D^{+}}$and its $B^{+}$-meson counterpart $f_{B^{+}}$are critical parameters of heavy-flavor physics. In $B$-meson physics, the $B^{0} \bar{B}^{0}$ mixing parameter $x_{B}=$ $\Delta M_{B} / \Gamma_{B}$ can be well measured, where $\Delta M_{B}$ and $\Gamma_{B}$ are the mass difference between the two neutral $B$-meson eigenstates and the mean neutral $B$-meson total width, respectively. In the SM, assuming the CKM matrix element $\left|V_{\mathrm{tb}}\right|=1$ the $x_{B}$ is given by

$$
x_{B}=\tau_{B} \frac{G_{F}^{2} M_{W}^{2}}{6 \pi} \eta_{B} S\left(x_{t}\right) M_{B} f_{B} \sqrt{B_{B}}\left|V_{\mathrm{td}}\right|^{2},
$$

where $B_{B}$ is the corresponding "bag parameter" and $\eta_{B} S\left(x_{t}\right)$ is perturbatively known [3]. Since $x_{B}$ is the theoretically and experimentally most accessible quantity, a reliable and precise determination of $f_{B^{+}}$is important for extracting $\left|V_{\mathrm{td}}\right|$. However, it is currently not possible to measure $f_{B^{+}}$directly from $B^{+}$leptonic decays with the required precision [4], so, theoretical calculations of $f_{B^{+}}$ have to be used in the determination of $\left|V_{\mathrm{td}}\right|$. In current lattice QCD (LQCD) calculations, the ratio $f_{D^{+}} / f_{B^{+}}$is determined with a significantly better precision than the individual quantities themselves. Thus, a precise measurement of $f_{D^{+}}$can be used to validate the LQCD calculation and subsequently be used in conjunction with the LQCD value for $f_{D^{+}} / f_{B^{+}}$to make a precise estimate of $f_{B^{+}}$. In turn, the resulting $f_{B^{+}}$value can be used to improve the precision of $\left|V_{\mathrm{td}}\right|$ determined from the measured $B^{0} \bar{B}^{0}$ mixing strength.

Measurements of $\left|V_{\mathrm{cd}}\right|$ have historically been based on measured branching fractions for semileptonic $D \rightarrow \pi \ell^{+} \nu_{\ell}$ decays and on measurements of charm production cross sections in neutrino and antineutrino interactions. However, extracting $\left|V_{\text {cd }}\right|$ from exclusive semileptonic decay rates requires a knowledge of the relevant hadronic form factor, which can have theoretical uncertainties that are about $11 \%$; the uncertainty of $\left|V_{\mathrm{cd}}\right|$ determined from neutrino and antineutrino cross sections is about 4.8\% [2]. A recent unquenched LQCD calculation of $f_{D^{+}}$claims a precision of about $2 \%$ [5] and provides an opportunity to improve the measured value of $\left|V_{\mathrm{cd}}\right|$ using an improved $D^{+} \rightarrow \mu^{+} \nu_{\mu}$ branching fraction determination.

In this paper we report measurements of the branching fraction for $D^{+} \rightarrow \mu^{+} \nu_{\mu}$ decay and the product of $f_{D^{+}}$and $\left|V_{\text {cd }}\right|$ based on analysis of $2.92 \mathrm{fb}^{-1}$ of data taken at $\sqrt{s}=$ $3.773 \mathrm{GeV}$ with the BESIII detector [6,7]. Using this measured $f_{D^{+}}\left|V_{\text {cd }}\right|$ together with the CKM matrix element $\left|V_{\text {cd }}\right|$, we determine the pseudoscalar decay constant $f_{D^{+}}$. Alternatively, using the measured $f_{D^{+}}\left|V_{\mathrm{cd}}\right|$ in conjunction with a lattice QCD prediction for $f_{D^{+}}$, we determine the CKM matrix element $\left|V_{\text {cd }}\right|$. This more accurate determination of $\left|V_{\mathrm{cd}}\right|$ and improved determination of $\left|V_{\mathrm{td}}\right|$ would improve the stringency of unitarity constraints on the CKM matrix and provide an improved test of the SM.

The BESIII [6] detector is a cylindrical detector with a solid-angle coverage of $93 \%$ of $4 \pi$ that operates at the BEPCII $e^{+} e^{-}$collider [6]. It consists of several main components. A 43-layer main drift chamber (MDC) which surrounds the beam pipe performs precise determinations of charged-particle trajectories and provides ionization energy loss $(d E / d x)$ measurements that are used for charged-particle identification. An array of time-of-flight counters (TOF) is located radially outside of the MDC and provides additional charged-particle identification information. The time resolution of the TOF system is $80 \mathrm{ps}$ (110 ps) in the barrel (end-cap) regions, corresponding better than $2 \sigma K / \pi$ separation for momentum below about $1 \mathrm{GeV} / \mathrm{c}$. The solid-angle coverage of the barrel TOF is $|\cos \theta|<0.83$, while that of the end cap is $0.85<$ $|\cos \theta|<0.95$, where $\theta$ is the polar angle of the coverage. A CsI(Tl) electromagnetic calorimeter (EMC) surrounds the TOF and is used to measure the energies of photons and electrons. The angular coverage of the barrel EMC is $|\cos \theta|<0.82$. The two end caps cover $0.83<|\cos \theta|<$ 0.93 . A solenoidal superconducting magnet located outside the EMC provides a $1 \mathrm{~T}$ magnetic field in the central tracking region of the detector. The iron flux return of the magnet is instrumented with $1600 \mathrm{~m}^{2}$ of resistive plate muon counters (MUC) arranged in nine layers in the barrel and eight layers in the end caps that are used to identify muons with momentum greater than $500 \mathrm{MeV} / \mathrm{c}$.

The center-of-mass energy of $3.773 \mathrm{GeV}$ corresponds to the peak of the $\psi(3770)$ resonance, which decays predominantly into $D \bar{D}$ meson pairs [2]. In events where a $\bar{D}$ meson is fully reconstructed, the remaining particles must all be decay products of the accompanying $D$ meson. In the following, the reconstructed meson is called the tagged $\bar{D}$. In a tagged $D^{-}$ data sample, events where the recoiling $D^{+}$decays to $\mu^{+} \nu_{\mu}$ can be cleanly isolated and used to provide a measurement of the absolute branching fraction $B\left(D^{+} \rightarrow \mu^{+} \nu_{\mu}\right)$.

Tagged $D^{-}$mesons are reconstructed in nine decay modes: $K^{+} \pi^{-} \pi^{-}, K_{S}^{0} \pi^{-}, K_{S}^{0} K^{-}, K^{+} K^{-} \pi^{-}, K^{+} \pi^{-} \pi^{-} \pi^{0}$, 
$\pi^{+} \pi^{-} \pi^{-}, K_{S}^{0} \pi^{-} \pi^{0}, K^{+} \pi^{-} \pi^{-} \pi^{-} \pi^{+}$, and $K_{S}^{0} \pi^{-} \pi^{-} \pi^{+}$. Events that contain at least three reconstructed charged tracks with good helix fits and $|\cos \theta|<0.93$ are selected, where $\theta$ is the polar angle of the charged tracks with respect to the beam direction. All charged tracks other than those from $K_{S}^{0}$ decays are required to have a distance of closest approach to the average $e^{+} e^{-}$interaction point that is less than $1.0 \mathrm{~cm}$ in the plane perpendicular to the beam and less than $15.0 \mathrm{~cm}$ along the beam direction. These charged tracks are then constrained to have a common vertex. The TOF and $d E / d x$ measurements are combined to form confidence levels for pion $\left(C L_{\pi}\right)$ and kaon $\left(C L_{K}\right)$ particle identification hypotheses. In this analysis pion (kaon) identification requires $C L_{\pi}>C L_{K} \quad\left(C L_{K}>C L_{\pi}\right)$ for tracks with momentum $p<0.75 \mathrm{GeV} / \mathrm{c}$, and $C L_{\pi}>$ $0.1 \%\left(C L_{K}>0.1 \%\right)$ for $p>0.75 \mathrm{GeV} / \mathrm{c}$.

For the selection of photons from $\pi^{0} \rightarrow \gamma \gamma$ decays, the deposited energy of a neutral cluster in the EMC is required to be greater than 25 (50) $\mathrm{MeV}$ if the crystal with the maximum deposited energy in that cluster is in the barrel (end-cap) region [6]. In addition, information about the EMC cluster hit time is used to suppress electronic noise and energy deposits unrelated to the event. In order to reduce backgrounds, the angle between the photon candidate and the nearest charged track is required to be greater than $10^{\circ}$. A one-constraint (1C) kinematic fit is used to constrain the invariant mass of $\gamma \gamma$ pairs to the mass of the $\pi^{0}$ meson in order to reduce combinatorial backgrounds. If the 1C kinematic fit converges with $\chi^{2}<100$, the pair is considered as a candidate $\pi^{0} \rightarrow \gamma \gamma$ decay.

We detect $K_{S}^{0}$ mesons that decay to a $\pi^{+} \pi^{-}$pair. A vertex fit is performed on two oppositely charged tracks that are assumed to be pions. If the vertex fit is successful and the invariant mass of the $\pi^{+} \pi^{-}$is in the range between 0.485 and $0.515 \mathrm{GeV} / \mathrm{c}^{2}$, the $\pi^{+} \pi^{-}$pair is taken as a candidate $K_{S}^{0}$ meson.

Tagged $D^{-}$mesons are identified by their beam-energyconstrained mass $M_{\mathrm{BC}}$ :

$$
M_{\mathrm{BC}}=\sqrt{E_{\text {beam }}^{2}-\left|\vec{p}_{m K n \pi}\right|^{2}},
$$

where $m$ and $n(m=0,1,2 ; n=0,1,2,3$, or 4) denotes the numbers of kaons and pions in the tagged $D^{-}$decay mode being considered, $E_{\text {beam }}$ is the beam energy, and $\left|\vec{p}_{m K n \pi}\right|$ is the magnitude of the three-momentum of the $m K n \pi$ system. In addition, the absolute value of the difference between the beam energy and the sum of the measured energies of the $m K n \pi$ combination is required to be within approximately $2.5 \sigma_{E_{m K n \pi}}$ of zero, where $\sigma_{E_{m K n \pi}}$ is the decay-mode-dependent standard deviation of the energy of the $m K n \pi$ system.

The $M_{\mathrm{BC}}$ distributions for the nine $D^{-}$tag modes are shown in Fig. 1. A maximum likelihood fit is used to obtain the number of tagged $D^{-}$events for each of the nine modes.
We use the Monte Carlo simulated signal shape convolved with a double-Gaussian resolution function to represent the beam-energy-constrained mass signal for the $D^{-}$daughter particles, and an ARGUS function [8] multiplied by a thirdorder polynomial [9] to describe the background shape to fit the $M_{\mathrm{BC}}$ distributions. In the fits all parameters of the double-Gaussian function, the ARGUS function, and the polynomial function are left free. We identify tagged $D^{-}$ candidates as combinations with $M_{\mathrm{BC}}$ within the range given by two red dashed lines in each figure. This requirement reduces the number of signal events by about $2 \%$ and keeps a total of $1703054 \pm 3405$ tagged $D^{-}$ mesons $\left(N_{D_{\text {tag }}^{-}}\right)$.

Candidate $D^{+} \rightarrow \mu^{+} \nu_{\mu}$ events are selected from the remaining charged tracks in the system recoiling against the tagged $D^{-}$-meson candidates by requiring that there be only one good positively charged track that is identified as a $\mu^{+}$. In BESIII, a $\mu^{+}$can be identified by its transit distance in the MUC, since charged hadrons (pions or kaons) undergo strong interactions with the absorber material and stop before penetrating very far into the MUC. In addition, in candidate $D^{+} \rightarrow \mu^{+} \nu_{\mu}$ events the maximum energy $E_{\gamma \max }$ of any extra good photon in the EMC is required to be less than $300 \mathrm{MeV}$.

Since there is only a single missing neutrino in $D^{+} \rightarrow$ $\mu^{+} \nu_{\mu}$ events, we require that the missing energy $E_{\text {miss }}$ and momentum $\vec{p}_{\text {miss }}$ are such that the value of the missing mass squared $M_{\text {miss }}^{2}$ is consistent with zero, where $M_{\text {miss }}^{2}$ is defined as

$$
M_{\text {miss }}^{2}=\left(E_{\text {beam }}-E_{\mu^{+}}\right)^{2}-\left(-\vec{p}_{D_{\text {tag }}^{-}}-\vec{p}_{\mu^{+}}\right)^{2} .
$$
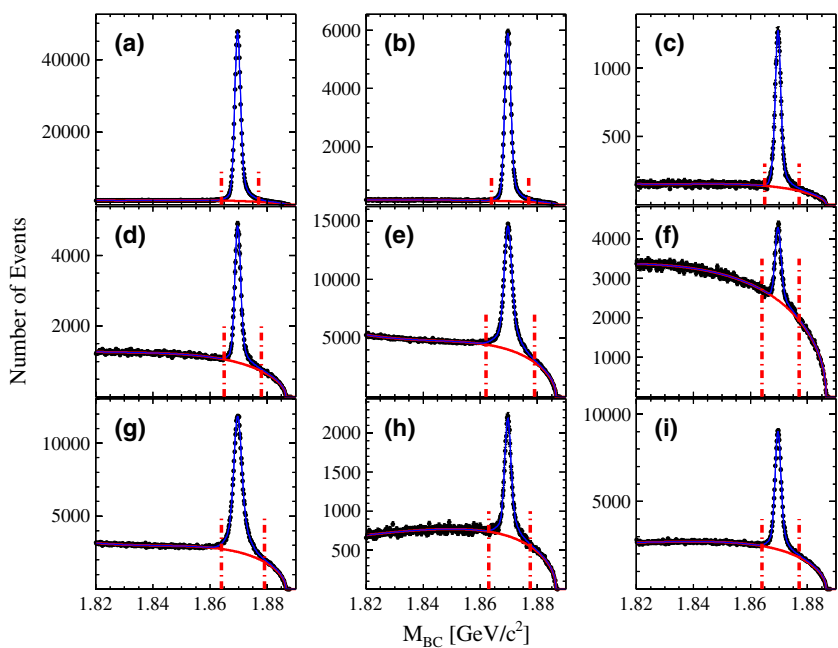

FIG. 1 (color online). The beam-energy-constrained mass distributions for the different $m K n \pi$ tagged mode combinations, where (a) $K^{+} \pi^{-} \pi^{-}$, (b) $K_{S}^{0} \pi^{-}$, (c) $K_{S}^{0} K^{-}$, (d) $K^{+} K^{-} \pi^{-}$, (e) $K^{+} \pi^{-} \pi^{-} \pi^{0}$, (f) $\pi^{+} \pi^{-} \pi^{-}$, (g) $K_{S}^{0} \pi^{-} \pi^{0}$, (h) $K^{+} \pi^{-} \pi^{-} \pi^{-} \pi^{+}$, and (i) $K_{S}^{0} \pi^{-} \pi^{-} \pi^{+}$; the two vertical dashed red lines show the tagged $D^{-}$mass region. 
Here $E_{\mu^{+}}$and $\vec{p}_{\mu^{+}}$are the energy and three-momentum of the $\mu^{+}$, respectively, and $\vec{p}_{D_{\text {tag }}^{-}}$is the three-momentum of the tagged $D^{-}$candidate. Figure 2 shows the $M_{\text {miss }}^{2}$ distribution for selected single $\mu^{+}$candidates. There are 451 candidate $D^{+} \rightarrow \mu^{+} \nu_{\mu}$ events in the $\left|M_{\text {miss }}^{2}\right|<0.12 \mathrm{GeV}^{2} / \mathrm{c}^{4}$ signal region as shown with two red arrows. The events that peak near $M_{\text {miss }}^{2} \simeq 0.25 \mathrm{GeV}^{2} / \mathrm{c}^{4}$ are primarily from $D^{+} \rightarrow$ $K_{L}^{0} \pi^{+}$decays, where the $K_{L}^{0}$ is undetected.

To check the Monte Carlo simulation, we compare the $M_{\text {miss }}^{2}$ distribution for $D^{+} \rightarrow K_{S}^{0} \pi^{+}$from the data with that from Monte Carlo simulated events, where the $K_{S}^{0}$ is missing in the calculation of $M_{\text {miss }}^{2}$. We select $D^{+} \rightarrow$ $K_{S}^{0} \pi^{+}$events with the same requirements as these used in selection of $D^{+} \rightarrow \mu^{+} \nu_{\mu}$, but require an additional $K_{S}^{0}$. We find that the $M_{\text {miss }}^{2}$ resolution for the data to be 1.194 times wider than that for the simulated events. To account for this difference, we scale the $M_{\text {miss }}^{2}$ resolution of simulated events by a factor of 1.194 when looking for $D^{+} \rightarrow \mu^{+} \nu_{\mu}$ signal and estimating numbers of peaking background events, such as $D^{+} \rightarrow K_{L}^{0} \pi^{+}$and $D^{+} \rightarrow \pi^{+} \pi^{0}$ decays (see below and see Fig. 2).

The numbers of the background events from $D^{+} \rightarrow$ $K_{L}^{0} \pi^{+}$and $D^{+} \rightarrow \pi^{+} \pi^{0}$, as well as $D^{+} \rightarrow \tau^{+} \nu_{\tau}$, are estimated by analyzing Monte Carlo samples that are 10 times larger than the data. The input branching fractions for $D^{+} \rightarrow K_{L}^{0} \pi^{+}$and $D^{+} \rightarrow \pi^{+} \pi^{0}$ are from Ref. [2]. For estimation of the backgrounds from $D^{+} \rightarrow \tau^{+} \nu_{\tau}$ decay, we use branching fraction $B\left(D^{+} \rightarrow \tau^{+} \nu_{\tau}\right)=$ $2.67 \times B\left(D^{+} \rightarrow \mu^{+} \nu_{\mu}\right)$, where $B\left(D^{+} \rightarrow \mu^{+} \nu_{\mu}\right)$ is quoted from Ref. [10] and 2.67 is expected by the SM.

The backgrounds from other $D$ decays are corrected considering the difference in the numbers of events from

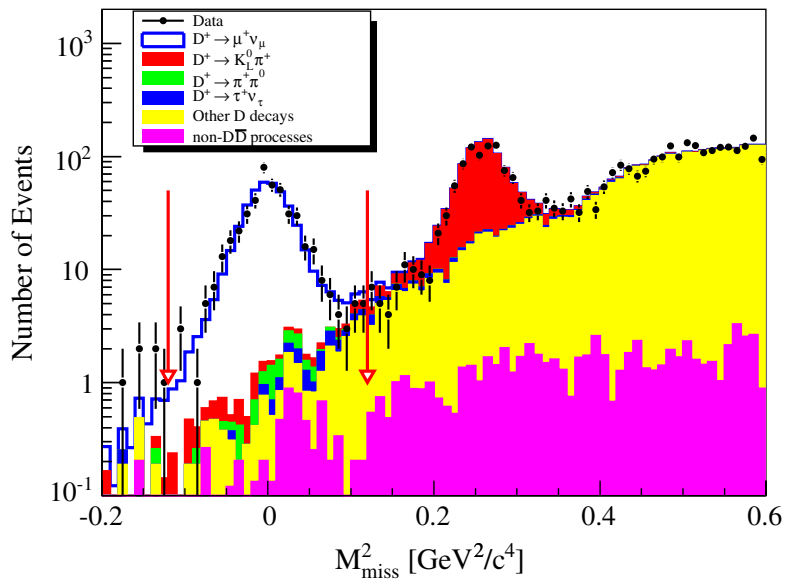

FIG. 2 (color online). The $M_{\text {miss }}^{2}$ distribution for selected single $\mu^{+}$candidates, where dots with error bars indicate the data, the opened histogram is for Monte Carlo simulated signal events of $D^{+} \rightarrow \mu^{+} \nu_{\mu}$ decays, and the hatched histograms are for the simulated backgrounds from $D^{+} \rightarrow K_{L}^{0} \pi^{+}$(red), $D^{+} \rightarrow \pi^{0} \pi^{+}$ (green), $D^{+} \rightarrow \tau^{+} \nu_{\tau}$ (blue), all other $D$-meson decays (yellow), and non- $D \bar{D}$ processes (pink). the data and simulated events in the range from 0.15 to $0.60 \mathrm{GeV}^{2} / \mathrm{c}^{4}$. Other background events are from $e^{+} e^{-} \rightarrow \gamma_{\mathrm{ISR}} \psi(3686), \quad e^{+} e^{-} \rightarrow \gamma_{\mathrm{ISR}} J / \psi, \quad$ where $\gamma_{\mathrm{ISR}}$ denotes the photon produced due to initial state radiation, $e^{+} e^{-} \rightarrow \mathrm{q} \overline{\mathrm{q}}(\mathrm{q}=\mathrm{u}, \mathrm{d}$, or $\mathrm{s}), e^{+} e^{-} \rightarrow \tau^{+} \tau^{-}$, and $\psi(3770) \rightarrow$ non- $D \bar{D}$ decays that satisfy the event-selection criteria of purely leptonic decays. The numbers of these background events are estimated by analyzing Monte Carlo samples of each of the above-listed processes, which are about 10 times more than the data. After normalizing these numbers of background events from the Monte Carlo samples to the data, we expect that there are $42.0 \pm 2.3$ background events, where the errors reflect the Monte Carlo statistics, uncertainties in the branching fractions, and/or production cross sections for the background channels.

After subtracting the number of background events, $409.0 \pm 21.2 \pm 2.3$ signal events $\left(N_{\text {sig }}^{\text {net }}\right)$ for $D^{+} \rightarrow \mu^{+} \nu_{\mu}$ remain, where the first error is statistical and the second is the systematic associated with the uncertainty of the background estimate. The weighted overall efficiency for detecting $D^{+} \rightarrow \mu^{+} \nu_{\mu}$ decays is determined to be $\epsilon=$ $0.6403 \pm 0.0012$ by analyzing Monte Carlo simulated events for $D^{+} \rightarrow \mu^{+} \nu_{\mu}$ in each tagged $D^{-}$mode; here the error is due to Monte Carlo statistics. Final state radiation is included in the Monte Carlo simulation.

Inserting $N_{D_{\text {tag }}^{-}}, N_{\text {sig }}^{\text {net }}$, and $\epsilon$ into

$$
B\left(D^{+} \rightarrow \mu^{+} \nu_{\mu}\right)=\frac{N_{\mathrm{sig}}^{\mathrm{net}}}{N_{D_{\mathrm{tag}}^{-}} \times \epsilon}
$$

and subtracting from the signal a $1.0 \%$ contribution coming from $D^{+} \rightarrow \gamma D^{*+} \rightarrow \gamma \mu^{+} \nu_{\mu}[10,11]$, in which $D^{*+}$ is a virtual vector or axial-vector meson, yields

$$
B\left(D^{+} \rightarrow \mu^{+} \nu_{\mu}\right)=(3.71 \pm 0.19 \pm 0.06) \times 10^{-4},
$$

where the first error is statistical and the second systematic. This measured branching fraction is consistent within errors with those measured at BES-I [12], BES-II [13], and CLEO-c [10], but with the best precision.

The systematic uncertainty in the $D^{+} \rightarrow \mu^{+} \nu_{\mu}$ branching fraction determination includes seven contributions: (1) the uncertainty in the number of $D^{-}$tags $(0.5 \%)$, which contain the uncertainty in the fit to the $M_{\mathrm{BC}}$ distribution $(0.5 \%)$ and the difference in the fake $\pi^{0}$ rates between the data and the Monte Carlo events $(0.1 \%)$; (2) the uncertainty in $\mu$ tracking/identification $(0.1 \% / 0.8 \%)$ determined by comparing the $\mu$ tracking/identification efficiencies for data and Monte Carlo events, where the $\mu^{ \pm}$samples are from the copious $e^{+} e^{-} \rightarrow \gamma \mu^{+} \mu^{-}$process; (3) the uncertainty in the $E_{\gamma_{\max }}$ selection requirement $(0.1 \%)$ determined by comparing doubly tagged $D \bar{D}$ hadronic decay events in the data and Monte Carlo; (4) the uncertainty associated with the choice of the $M_{\text {miss }}^{2}$ signal window $(0.5 \%)$ determined from changes in the measured branching fractions using different 
signal window widths; (5) the uncertainty in the background estimate $(0.6 \%)$ due to Monte Carlo statistics of the simulated backgrounds and uncertainties in the branching fractions or the production cross sections for the background channels; (6) the uncertainty in efficiency $(0.2 \%)$ arising from the Monte Carlo statistics; (7) the uncertainty in the radiative correction $(1.0 \%)$, which we take to be $100 \%$ of its central value $[10,11]$. The total systematic error determined by adding all the component errors in quadrature is $1.6 \%$.

Inserting the measured branching fraction, $G_{F}$, the mass of the muon, the mass of the $D^{+}$meson, and the lifetime of the $D^{+}$meson [2] into Eq. (1) yields

$$
f_{D^{+}}\left|V_{\mathrm{cd}}\right|=(45.75 \pm 1.20 \pm 0.39) \mathrm{MeV}
$$

where the first error is statistical and the second systematic arising mainly from the uncertainties in the measured branching fraction $(1.6 \%)$ and the lifetime of the $D^{+}$ meson $(0.7 \%)$ [2]. The total systematic error is $0.9 \%$ for $f_{D^{+}}\left|V_{\mathrm{cd}}\right|$.

The decay constant $f_{D^{+}}$is obtained using as input the CKM matrix element $\left|V_{\text {cd }}\right|=0.22520 \pm 0.00065$ from the global fit in the SM [2]. Alternatively, $\left|V_{\text {cd }}\right|$ is determined using $f_{D^{+}}=207 \pm 4 \mathrm{MeV}$ from LQCD [5] as input. The results are

$$
f_{D^{+}}=(203.2 \pm 5.3 \pm 1.8) \mathrm{MeV}
$$

and

$$
\left|V_{\mathrm{cd}}\right|=0.2210 \pm 0.0058 \pm 0.0047
$$

where the first errors are statistical and the second systematic arising mainly from the uncertainties in the measured branching fraction $(1.6 \%)$, the CKM matrix element $\left|V_{\mathrm{cd}}\right|(0.3 \%), f_{D^{+}}(1.9 \%)$, and the lifetime of the $D^{+}$meson $(0.7 \%)$ [2]. The total systematic error is $0.9 \%$ for $f_{D^{+}}$and $2.1 \%$ for $\left|V_{\text {cd }}\right|$.

Our measured value for $B\left(D^{+} \rightarrow \mu^{+} \nu_{\mu}\right)$ has the best precision in the world to date. The value of $f_{D^{+}}$can be used to validate LQCD calculations of $f_{D^{+}}$, thereby producing a more reliable and precise prediction of $f_{B^{+}}$. This $f_{B^{+}}$value can in turn be used to improve the precision of the determination of $\left|V_{\mathrm{td}}\right|$, and the improved $\left|V_{\mathrm{cd}}\right|$ and $\left|V_{\mathrm{td}}\right|$ can be used for more stringent tests of the SM.

\section{ACKNOWLEDGMENTS}

The BESIII Collaboration thanks the staff of BEPCII and the computing center for their strong support. This work is supported in part by the Ministry of Science and Technology of China under Contracts No. 2009CB825204 and No. 2009CB825200; National Natural Science Foundation of China (NSFC) under Contracts No. 10625524, No. 10821063, No. 10825524, No. 10835001, No. 10935007, No. 11125525, and No. 11235011; Joint Funds of the National Natural Science Foundation of China under Contracts No. 11079008 and No. 11179007; the Chinese Academy of Sciences (CAS) Large-Scale Scientific Facility Program; CAS under Contracts No. KJCX2-YW-N29 and No. KJCX2-YW-N45; 100 Talents Program of CAS; German Research Foundation DFG under Contract No. Collaborative Research Center CRC-1044; Istituto Nazionale di Fisica Nucleare, Italy; Ministry of Development of Turkey under Contract No. DPT2006K-120470; U.S. Department of Energy under Contracts No. DE-FG02-04ER41291, No. DEFG02-05ER41374, No. DE-FG02-94ER40823, and No. DESC0010118; U.S. National Science Foundation; University of Groningen ( $\mathrm{RuG}$ ) and the Helmholtzzentrum für Schwerionenforschung GmbH (GSI), Darmstadt; WCU Program of National Research Foundation of Korea under Contract No. R32-2008-000-10155-0.
[1] D. Silverman and H. Yao, Phys. Rev. D 38, 214 (1988); J. L. Rosner, Phys. Rev. D 42, 3732 (1990); C. H. Chang and Y. Q. Chen, Phys. Rev. D 46, 3845 (1992); 49, 3399 (1994).

[2] J. Beringer et al. (Particle Data Group), Phys. Rev. D 86, 010001 (2012).

[3] See, for example, C. Bernard et al., arXiv:hep-ph/9709328.

[4] K. Hara et al. (Belle Collaboration), Phys. Rev. Lett. 110, 131801 (2013); B. Aubert et al. (BABAR Collaboration), Phys. Rev. D 81, 051101(R) (2010).

[5] E. Follana, C. Davies, G. Lepage, and J. Shigemitsu (HPQCD and UKQCD Collaborations), Phys. Rev. Lett. 100, 062002 (2008).

[6] M. Ablikim et al. (BESIII Collaboration), Nucl. Instrum. Methods Phys. Res., Sect. A 614, 345 (2010).
[7] M. Ablikim et al. (BESIII Collaboration), Chin. Phys. C 37, 123001 (2013).

[8] H. Albrecht et al. (ARGUS Collaboration), Phys. Lett. B 241, 278 (1990).

[9] M. Ablikim et al. (BES Collaboration), Phys. Lett. B 597, 39 (2004).

[10] B. I. Eisenstein et al. (CLEO Collaboration), Phys. Rev. D 78, 052003 (2008).

[11] G. Burdman, J. T. Goldman, and D. Wyler, Phys. Rev. D 51, 111 (1995).

[12] J. Z. Bai et al. (BES Collaboration), Phys. Lett. B 429, 188 (1998).

[13] M. Ablikim et al. (BES Collaboration), Phys. Lett. B 610, 183 (2005). 